Journal of Animal and Veterinary Advances 11 (15): 2756-2761, 2012

ISSN: $1680-5593$

(C) Medwell Journals, 2012

\title{
Development and Evaluation of a Reverse Transcription-Loop-Mediated Isothermal Amplification Method for Rapid Detection of Novel Duck Reovirus
}

\author{
${ }^{1,2}$ Zhao-Long Li, ${ }^{1,2}$ Shi-Long Chen, ${ }^{1,2}$ Shao-Ying Chen, ${ }^{1,2}$ Feng-Qian Lin, \\ ${ }^{1,2}$ Xiao-Xia Cheng, ${ }^{1,2}$ Shao Wang, ${ }^{1}$ Xiao-Li Zhu and ${ }^{1}$ Bin Jiang \\ ${ }^{1}$ Department of Animal Husbandry and Veterinary Medicine, \\ ${ }^{2}$ Department of Fujian Animal Diseases Control Technology Development Center, \\ Fujian Academy of Agriculture Sciences, Fuzhou, 350013 Fujian, China
}

\begin{abstract}
Novel Duck Reovirus (NDRV) is the major causative agent of the Duck Necrotic Hemorrhagic Hepatitis (DNHH), it causes huge economic losses to the duck industry in China. The affected ducks (including Muscovy, Mule, Peking ducks and Sheldrake) manifest the main clinical symptoms with hemorrhage and necrosis in the hepatitis which can cause high mortality in ducks. Develop a rapid and simple detection is the most efficient way for prevention and control the disease in short time. In this study, a Reverse Transcription Loop-mediated isothermal Amplification (RT-LAMP) assay was employed for detecting the disease. The sensitivity of RT-LAMP assay was 1000 folds greater and the detection limit was $2.8 \times 10^{-3} \mathrm{ng}$ RNA. In addition, the specificity results shown that it was no any cross-reaction with other duck's viruses. The RT-LAMP assay may be a good method for specific diagnosis of NDRV infection in fewer well-equipped laboratories, especially in farm or rural veterinary clinics.
\end{abstract}

Key words: Reverse Transcription Loop-mediated isothermal Amplification (RT-LAMP), Novel Duck reovirus (NDRV), detection, sensitivity, rapidity, specificity

\section{INTRODUCTION}

Duck Necrotic Hemorrhagic Hepatitis (DNHH) was outbreaks in 2005 (Chen and Lin, 2009) and rapidly spread around the duck-producing regions in China (Guangdong and Fujian). The infected ducks (including Muscovy, Mule, Peking ducks and Sheldrake) manifest some clinical symptom with hemorrhage and necrosis in the hepatitis (Chen and Cheng, 2010). The morbidity rate is from 5-20\% and mortality rates be closed in $20 \%$. Sometimes, the mortality rate for this disease was $>50 \%$, especially for duckling and colder weather (Wang and Cheng, 2010a, b). At present, there is no effective vaccine or antiviral therapy available for the $\mathrm{DNHH}$ disease. It has caused a huge economic loss for the duck-producing regions. The pathogen has been identified a Novel Duck Reovirus (NDRV) by electron microscopic observation, animal regression some physical chemical and biological characteristics and the analysis of nucleic acid segments (Chen and Lin, 2009; Chen and Cheng, 2010; Wang and Cheng, 2010a, b).

NDRV is a member of the Orthoreovirus genus of the family Reoviridae, share common physico-chemical properties and morphological characteristics (Engstrom,
1988; Touris-Otero et al., 2005). The genome consists of 10 segments of double-stranded RNA (dsRNA) including three large segments (L1-L3), three medium segments (M1-M3) and four small segments (S1-S4) (Kuntz-Simon et al., 2002; Kouwenhoven et al., 1988; Mukiibi-Muka and Jones, 1999; Palya and Glavits, 2003). In spite of being the family reoviridae, NDRV showed just approximately $70 \%$ amino acid sequence and nucleotide sequence identity to another reovirus. According to the highly variable sequences of the S3 and M3, ducks reovirus can be classified into Novel Duck Reovirus (NDRV), Muscovy Duck Reovirus (MDRV) and Avian Reovirus (ARV) (Wang and Cheng, 2010a, b).

In resent years, there has been an increasing method to diagnosis of NDRV such as virus isolation, RT-PCR assay and detection of virus-specific antibodies (Chen and Lin, 2009; Chen and Cheng, 2010; Wang and Cheng, 2010a, b). NDRV can be defined by virus isolation which always spends 2 weeks to ascertain the results. The detection of RT-PCR for NDRV has high sensitivities and specificity in the recently report but it requires high-precision instruments for the amplification or elaborate methods for the detection the products. The assays of virus-specific antibodies need serum sample

Corresponding Author: Zhao-long Li, Department of Animal Husbandry and Veterinary Medicine,

Fujian Academy of Agriculture Sciences, 247 Wusi Road, Fuzhou, 350013 Fujian, China

2756 
and especial equipment which are difficult to obtain the specific antibodies. Loop-mediated isothermal Amplification (LAMP) is a fast easy and reliable method which was first developed by Notomi et al. (2000). The LAMP method has high specificity and sensitivity that was easily performed by the isothermal condition such as water bath or block heater is a sufficient method for the DNA amplification (Xu and Zhang, 2009; Imai et al., 2006; Chen et al., 2008a, b). In addition, the results can be defined by visually under fluorescent dye. Presently, a number of Reverse Transcriptions Loop-mediated Isothermal Amplification (RT-LAMP) and LAMP tests have been used to detection human diseases, swine and other poultry virus diseases successfully (Xie et al., 2010; Komiyama et al., 2009; Lalande et al., 2011; Nagdev and Kashyap, 2011; Tao and Deng, 2011; Curtis and Rudolph, 2008; Deng et al., 2010; Li and Yue, 2004; Kuan et al., 2006; Kono et al., 2004). In this study, a very sensitive easy and less time-consuming RT-LAMP assay was established for diagnosis the NDRV in fewer well-equipped laboratories.

\section{MATERIALS AND METHODS}

Virus: NDRV (NP01-NP03) were isolated from the infected ducks in Fujian. Duck Hepatitis Virus (DHV) infectious Bursal Disease Virus (IBDV), Muscovy Duck Parvovirus (MDPV), Goose Parvovirus (GPV) Duck paramyxovirus (DPMV), Muscovy Duck Reovirus (MDRV) and Avian Reovirus ( ARV) were stocked at $80^{\circ} \mathrm{C}$ in the laboratory.

Designing of primers: The primers of RT-LAMP were designed from a relatively variable region on the $S 3$ gene of NDRV-NP03 strain genome position ( $S 3$ gene GenBank accession No. GQ888710). The target region of $500 \mathrm{bp}$ was selected for the aligned sequences. The detailed sequences of primers used for amplification of NDRVNP03 are shown in Table 1. Eight primers were designed by the primer explorer 4.0 software (http://primerexplorer. jp/elamp4.0.0/index.html) containing two outer primers (F3 and B3), four inner primers ( $\mathrm{FIP}=\mathrm{F} 1 \mathrm{c}+\mathrm{AAGCTT}+\mathrm{F} 2$ and $\mathrm{BIP}=\mathrm{B} 1+\mathrm{AAGCTT}+\mathrm{B} 2 \mathrm{c}$ ) and two loop primers (loop F and $\mathrm{B}$ ) that recognizing eight distinct regions on the $S 3$ gene. In addition, a pair of primers for RT-PCR amplification (named S1 and S2) was designed using the Primer Premier 5.0 Software based on the $S 3$ gene of NDRV-NP03. All primers were synthesized by Takara Biotechnology Co., Ltd. (Dalian, China).

DNA/RNA extraction: The genomic DNA/RNA was extracted from $200 \mu \mathrm{L}$ samples using a MiniBEST Viral RNA/DNA Extraction kit (Takara Biotechnology, Dalian, China), according to the protocol suggested by the manufacturer. The total RNA was stored at $-80^{\circ} \mathrm{C}$ until further use.

Optimization of the RT-LAMP conditions: The RT-LAMP was carried out in a $50 \mu \mathrm{L}$ mixture. The reaction system contained $2.5 \mu \mathrm{M}$ each of the primers FIP and BIP, $0.5 \mu \mathrm{M}$ each of the out primers $\mathrm{F} 3$ and $\mathrm{B} 3,0.5 \mu \mathrm{M}$ each LF and LB, $2 \mathrm{mM}$ each deoxynucleoside Triphosphate (dNTP), $20 \mathrm{mM}$ Tris/ $\mathrm{HCl}(\mathrm{pH} 8.8), 10 \mathrm{mM} \mathrm{KCl}, 10 \mathrm{mM}\left(\mathrm{NH}_{4}\right)_{2} \mathrm{SO}_{4}, 8 \mathrm{mM}$ $\mathrm{MgSO}_{4}, 0.1 \%$ Tween-20, $8 \mathrm{U}$ of the Bst DNA polymerase large fragment (New England Biolabs), $1 \mathrm{M}$ Betaine (SIGMA-ALDRICH, Germany), $0.2 \mathrm{mg} \mathrm{mL}{ }^{-1}$ Dimethyl Sulfoxide (DMSO from SIGMA, Germany), 2 u M-MLV reverse transcriptase (Promega, USA) and $4 \mu \mathrm{L}$ of target RNA, $1 \mu \mathrm{L}$ fluorescent regent. The reaction mixture was incubated at $60-68^{\circ} \mathrm{C}$ for $25-59 \mathrm{~min}$, respectively to gain the optimal temperature and time. Then, the reaction was terminated by heating at $80^{\circ} \mathrm{C}$ for $2 \mathrm{~min}$.

RT-PCR: The RT-PCR was performed by using the two primers targeting the $S 3$ gene of NDRV-NP03. The sequences of two primers were 5' ACCTCAGGATATCGCTGAAACT $3^{\prime}$ (sense) and $5^{\prime}$ CTCCATCCCTGCAGCACAT GTAAAG 3 ' (antisense). The cDNA of NDRV-NP03 was synthesized using the M-MLV reverse transcriptase at $42^{\circ} \mathrm{C}$ for $2 \mathrm{~h}$. PCR was used to amplify the $586 \mathrm{bp}$ gene of $S 3$.

The reaction mixtures were consisted of $2.5 \mu \mathrm{L}$ of $10 \times$ buffer, $2 \mu \mathrm{L}$ of dNTP, $1 \mu \mathrm{L}$ of each primer and $0.25 \mu \mathrm{L}$ of Ex Taq Hot Start (Takara Biotechnology, Dalian, China). The reaction was performed at $94^{\circ} \mathrm{C}$ for $5 \mathrm{~min}, 30$ cycles at $94^{\circ} \mathrm{C}$ for $45 \mathrm{sec}, 54^{\circ} \mathrm{C}$ for $45 \mathrm{sec}$ and $72^{\circ} \mathrm{C}$ for $1 \mathrm{~min}$ and

Table 1: RT-LAMP $S 3$ gene primer sets designed for rapid detection of novel ducks reovirus

\begin{tabular}{llcc}
\hline Primer name & Oligonucleotide sequence of the primers & Position in genome & Length of oligonucleotide (bp) $^{2}$ \\
Forward outer (F3) & TGCTTGCTGGAATTCGAGA & $37-56$ & 20 \\
Backward outer (B3) & CTCTGTAGTGCGACCCACC & $240-257$ & 19 \\
Forward Inner Primer (FIP) (F1c + AAGCTT + F2) & ACCACAGCACTGAGTGCAACAGTAA & $70-89$ & 25 \\
& TTGAAGAGTTCACACA & $111-132$ & 17 \\
Backward Inner Primer (BIP) (B1 + AAGCTT + B2c) & TGCCCCCCCTCTGATGGTAAC & $205-223$ & 22 \\
& GTCTCAGTACGATATTGC & $142-163$ & 18 \\
Forward Loop primer (FL) & GGACTGCAATAGTCCCAGCCGTTGC & $89-108$ & 25 \\
Backward Loop primer (BL) & GTTTTCCACATCACAAGTGTCA & $178-203$ & 22 \\
\hline
\end{tabular}

${ }^{a}$ Genome position (GenBank accession No. GQ888710) 
final extension at $72^{\circ} \mathrm{C}$ for $10 \mathrm{~min}$. Then, the amplified products were electrophoresed on $1.5 \%(\mathrm{w} / \mathrm{v})$ agarose gels $(90 \mathrm{~V}, 30 \mathrm{~min})$.

Sensitivity and specificity of RT-LAMP: The sensitivity of the RT-LAMP assay was performed using 10 fold serial dilutions of viral RNA from 280 to $2.8 \times 10^{-4} \mathrm{ng}$. The specificity of RT-LAMP assay was carried out by the cross-reactivity with other duck's pathogens such as DHV, IBD, MDPV, GPV, DPMV, MDRV and ARV.

Evaluation the RT-LAMP assay with clinical specimen: The applicability of the RT-LAMP assay for the clinical diagnosis of the NDRV-NP03 was validated with some specimens from the field isolation, amniotic fluid of NDRV-NP03 virus strain in embryonated chicken and duck eggs and cell-adapted of NDRV-NP03 virus strains. Forty samples were tested by the RT-LAMP and RT-PCR in order to evaluation the two assays which one was more adapted to identification and diagnosis the NDRV disease.

\section{RESULTS AND DISCUSSION}

Optimized reaction conditions of the RT-LAMP for NDRV: The RT-LAMP reaction has exhibited slightly larger amounts DNA amplified in a conventional water bathe for 40 at $63^{\circ} \mathrm{C}$ and the optimal amplification temperature is $63^{\circ} \mathrm{C}$ for 40 and the best reaction concentration of $\mathrm{Mg}^{2+}$ at $6 \mathrm{mM}$.

Sensitivity and specificity of RT-LAMP: The analytical sensitivities of the RT-LAMP assays were evaluated against a 10 fold serial dilution of NDRV-NP03 RNA. The result indicated that the detection limit of RT-LAMP was $2.8 \times 10^{-3}$ ng of RNA (Fig. 1a and b) but the RT-PCR assay limit was $2.8 \mathrm{ng}$ with the same serial dilution of NDRVNP03 RNA (Fig. 1c). Compared with RT-PCR for the detection of NDRV-NP03 RNA, the RT-LAMP assay showed 1000 fold higher sensitivity.

To evaluate the specificities of the RT-LAMP for NDRV RNA, another relevance duck's pathogens were detected by RT-LAMP. As a result, no cross-reactivity was observed with RNA from other duck's pathogens (Fig. 2a and b).

The products of RT-LAMP were digested with the restriction enzyme Hind III (Fig. 2c) after $1 \mathrm{~h}$ digestion, obtained one $200 \mathrm{bp}$ fragment which is in agreement with the previous forecast result.

Evaluation of NDRV RT-LAMP for clinical specimens: In here 60 NDRV RNA specimens were used to evaluate the

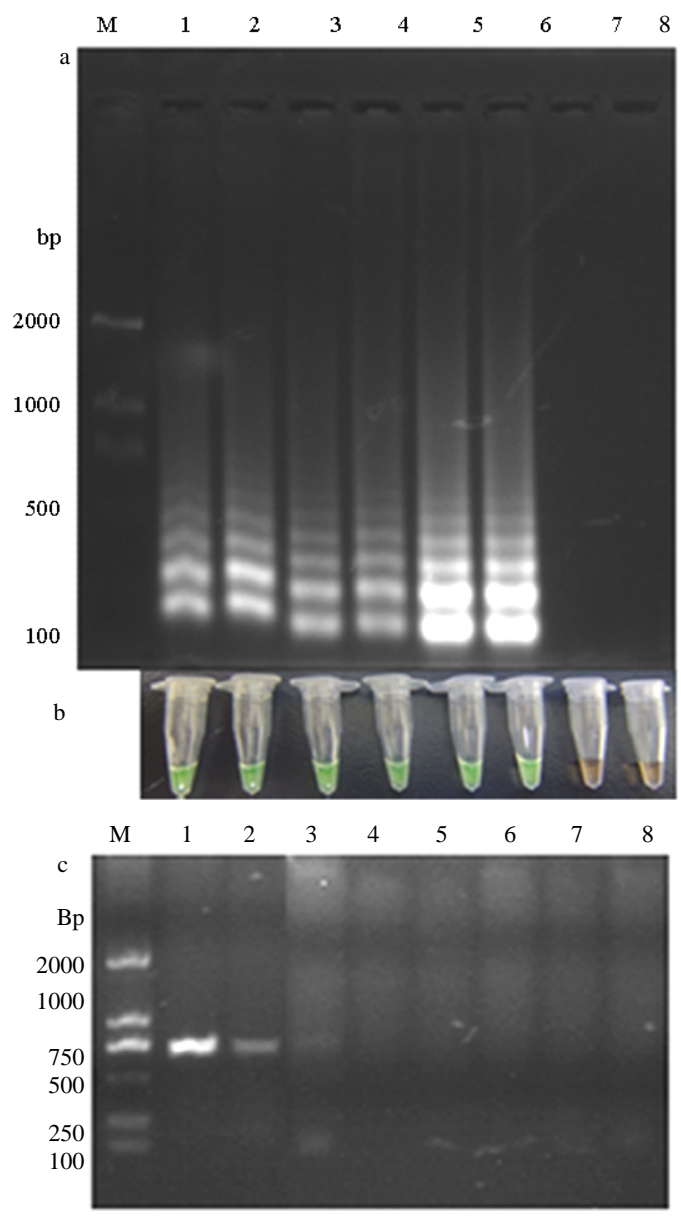

Fig. 1: Comparative the sensitivity of RT-LAMP and RT-PCR methods. RNA standards in vitro transcribed and serially diluted were subjected to RT-LAMP and RT-PCR detection; a) The products of RT-LAMP were tested by gel electrophoresis; b) The products of RT-LAMP were tested by UV light; c) The products of RT-PCR were tested by gel electrophoresis. Lanes $\mathrm{M}$, DNA marker DL2000; 1-7: $2.8 \times 10^{2}, 2.8 \times 10^{1}, 2.8 \times 10^{0}, 2.8 \times 10^{-1}$, $2.8 \times 10^{-2}, 2.8 \times 10^{-3}$ and $2.8 \times 10^{4} \mathrm{ng} \mathrm{RNA}$ of the NDRV, respectively; 8, DEPC-treated water

feasibility of RT-LAMP for detecting NDRV in clinical diagnosis. In parallel, conventional RT-PCR were also performed. Researchers found that 52 specimens in the RT-LAMP were defined positive but 48 samples from 60 specimens in the RT-PCR assay were detected positive (Table 2). The correlation between RT-LAMP and conventional RT-PCR was $86 \%$.

NDRV is a new member of reoviridae. It has produced serious damage in duck's hepatitis and most of the infected ducks died rapidly, especially the infected 


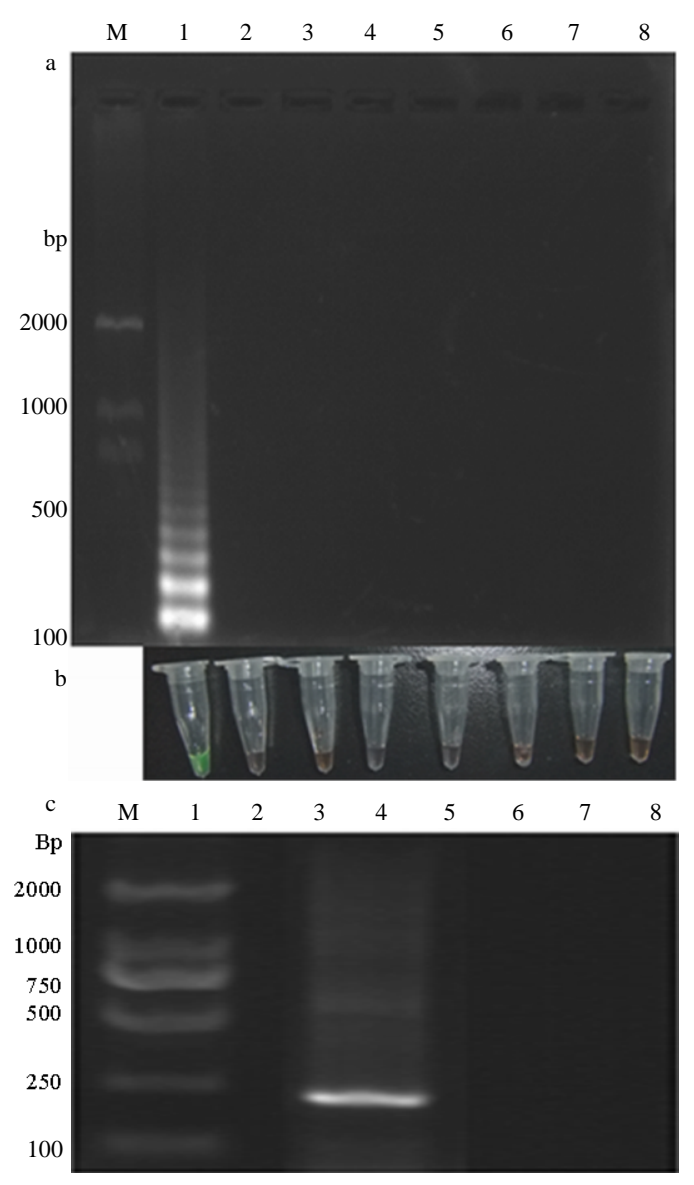

Fig. 2: The specificity of RT-LAMP assay for NDRV was confirmed by gel electrophoresis, visually inspection and the restriction enzyme HindIII; a) Agarose gel illustrating the specificity of the RT-LAMP assay among different species; $b$ ) The specific of RT-LAMP was examined by visually inspection. Lanes M, DNA marker DL2000; 1-9: The RNA of NDRV-NP03, Duck Hepatitis Virus (DHV), Infectious Bursal Disease (IBD), Muscovy Duck Parvovirus (MDPV), Goose Parvovirus (GPV), Duck Paramyxovirus (DPMV), Muscovl Duck Reoviurs (MDRV) and Avian Reovirus $(\mathrm{ARV})$; c) Verification of the specific RT-LAMP product of NDRV-NP03 by HindIII Digestion. Lanes M, DNA marker DL2000; 1, 2: The products of NDRV-NP03 RT-LAMP assay digested by HindIII

ducklings, nearly all died in a whole day. In the initial stages of the disease, the infected ducks appear the necrotic hemorrhagic hepatitis which was similar to MDRV disease. Thus, the NDRV disease often was misdiagnosed as MDRV .At the present because of the lack of a sensitive, reliable, rapid and simple diagnosis
Table 2: Comparison of RT-LAMP and RT-PCR assays in clinical specimens

\begin{tabular}{lccc}
\hline & No. of samples with indicated result by (assay) & \\
& - & RT-PCR & $\begin{array}{c}\text { Correlation } \\
(\%)\end{array}$ \\
Results & RT-LAMP & 43 & 86 \\
Positive & 50 & 7 & - \\
Negative & 0 & 50 & - \\
Total & 50 & &
\end{tabular}

tool in the basic place, it is a difficulty to control the NDRV disease in resent years. As a result, it has caused tremendous economic loss for the farm in China. Therefore, there is great requiring a rapid and simple diagnostic method for detection of NDRV to prevent and control the disease. In this study, researchers developed a one step RT-LAMP assay for detection of NDRV from clinical specimens. As shown in results, the sensitivity of RT-LAMP for the NDRV specimens is $2.8 \times 10^{-3} \mathrm{ng}$ which was the 1000 fold than the conventional RT-PCR. Because the Bst DNA polymerase has high amplification efficiency at $60-67^{\circ} \mathrm{C}$, the large numbers amplification of RT-LAMP was achieved within $40 \mathrm{~min}$ using water bathe at $63^{\circ} \mathrm{C}$. The result demonstrated that the RT-LAMP detection is highly sensitive than the RT-PCR with very low viral titers.

In addition, the 6 primers of RT-LAMP assay that recognizing the eight distinct regions of $S 3$ gene on NDRV genomes which prevent cross-reactions. In this study, the specific for clinical sample detection was high in comparisons with a conventional RT-PCR and no cross-reaction with other ducks pathogen was found in this study such as DHV, BDD, MDPV, GPV, DPMV, MDRV and ARV.

The RT-LAMP assay is a simple and rapid diagnostic tool. The reaction was carried out in a single tube by mixing the primers, reaction buffer, reverse transcriptase and Bst DNA polymerase and incubating the mixture at $63^{\circ} \mathrm{C}$ for 40 minimum in a regular water bath. Compared to RT-PCR, virus isolation and other detection, the RT-LAMP not needs expensive reagents, special operation and sophisticated instrumentation.

The attractive feature of the RT-LAMP for NDRV diseases, the products of RT-LAMP could be clearly defined by naked-eye after SYBR Green I dye-mediated, the visual detection using the RT-LAMP for NDRV diseases is faster and easier than the RT-PCR. Therefore, the method is a potential field usable tool for diagnostic and for use in NDRV control programs.

In clinical specimens, 60 specimens were analyzed by the RT-LAMP and RT-PCR assay. About 52 samples tested positive by the RT-LAMP, the results be agreement with the virus isolation. However, just 48 samples tested positive by RT-PCR. This may be shown that the RT-LAMP assay is a reliable detects method in basic place. 


\section{CONCLUSION}

This study describes that the single-tube and one step RT-LAMP assay is a reliable, rapid, easy, simple and sensitive to the diagnosis of NDRV. This has potential usefulness for clinical diagnosis and applications in the field.

\section{ACKNOWLEDGEMENTS}

The study was financially supported by the National High-Tech Research and Development Projects (863) (Grant No. 2011AA10A209), National Natural Science Foundation of China (Grant No. 31172334), Fujian Public Project (Grant No. 2010R1 101003-5) and Fujian Academy of Agricultural Sciences Innovation Team (Grant No. STIF-Y02).

\section{REFERENCES}

Chen, C.S. and F.Q. Lin, 2009. The primary study of pathogen of duck hemorrhagic-necrotic hepatitis. China Agric. Sci. Bull., 25: 28-31.

Chen, C.S. and X.X. Cheng, 2010. The study on the pathogenicity of new type duck reovirus. J. Northwest Univ. Nat. Sci., 38: 14-19.

Chen, H.T., J. Zhang, D.H. Sun, L.N. Ma, X.T. Liu, K. Quan and Y.S. Liu, 2008. Reverse transcription loop-mediated isothermal amplification for the detection of highly pathogenic porcine reproductive and respiratory syndrome virus. J. Virol. Methods, 153: 266-268.

Chen, H.T., J. Zhang, D.H. Sun, L.N. Ma, X.T. Liu, X.P. Cai and Y.S. Liu, 2008. Development of reverse transcription loop-mediated isothermal amplification for rapid detection of $\mathrm{H} 9$ avian influenza virus. J. Virol. Methods, 151: 200-203.

Curtis, K.A. and D.L. Rudolph, 2008. Rapid detection of HIV-1 by reverse-transcription, loop-mediated isothermal amplification (RT-LAMP). J. Virol. Methods, 151: 264-270.

Deng, X., X. Qi, Y. Gao, Y. Wang and L. Qin et al., 2010. Development of a loop-mediated isothermal amplification method for rapid detection of reticuloendotheliosis virus. J. Virol. Methods, 168: 82-86.

Engstrom, B.E., 1988. Blue wing disease of chickens: Isolation of avian reovirus and chicken anaemia agent. Avi. Pathol., 17: 23-32.
Imai, M., A. Ninomiya, H. Minekawa, T. Notomi, T. Ishizaki, M. Tashiro and T. Odagiri, 2006. Development of H5-RT-LAMP (loop-mediated isothermal amplification) system for rapid diagnosis of H5 avian influenza virus infection. Vaccine, 24: 6679-6682.

Komiyama, C., K. Suzuki, Y. Miura and H. Sentsui, 2009. Development of loop-mediated isothermal amplification method for diagnosis of bovine leukemia virus infection. J. Virol. Methods, 157: 175-179.

Kono, T., R. Savan, M. Sakai and T. Itami, 2004. Detection of white spot syndrome virus in shrimp by loopmediated isothermal amplification. J. Virol. Methods, 115: 59-65.

Kouwenhoven, B., M. Vertommen and E. Goren, 1988. Investigations into the role of reovirus in the malabsorption syndrome. Avi. Pathol., 17: 879-892.

Kuan, C.P., M.T. Wu, Y.L. Lu and H.C. Huang, 2006. Rapid detection of squash leaf curl virus by loop-mediated isothermal amplification. J. Virol. Methods, 169: 61-65.

Kuntz-Simon, G., G. le Gall-Recule, C. de Boisseson and V. Jestin, 2002. Muscovy duck reovirus rC protein is atypically encoded by thesmallest genome segment. J. Gen. Virol., 83: 1189-1200.

Lalande, V., L. Barrault, S. Wadel, C. Eckert, J.C. Petit and F. Barbut, 2011. Evaluation of a loop-mediated isothermal amplification assay for diagnosis of clostridium difficile infections. J. Clin. Microbiol., 49: 2714-2716.

Li, Q. and Z. Yue, 2004. Development and evaluation of a loop-mediated isothermal amplification assay for rapid detection of lymphocystis disease virus. J. Virol. Methods, 163: 378-384.

Mukiibi-Muka, G. and R.C. Jones, 1999. Local and systemic IgA and IgG responses of chicks to avian reoviruses: Effects of age of chick, route of infection and virus strain. Avi. Pathol., 28: 54-60.

Nagdev, K.J. and R.S. Kashyap, 2011. Loop-mediated isothermal amplification for rapid and reliable diagnosis of tuberculous meningitis. J. Clin. Microbiol., 49: 1861-1865.

Notomi, T., H. Okayama, H. Masubuchi, T. Yonekawa, K. Watanabe, N. Amino and T. Hase, 2000. Loopmediated isothermal amplification of DNA. Nucl. Acids Res., Vol. 28. 10.1093/nar/28.12.e63

Palya, V. and R. Glavits, 2003. Reovirus identified as cause of disease in young geese. Avi. Pathol., 32: 129-138. 
Tao, J.L. and Y.Q. Deng, 2011. Development and evaluation of a reversetranscription-loop-mediated isothermal amplification assay for rapid detection of enterovirus. J. Clin. Microbiol., 49: 870-874.

Touris-Otero, F., J.M. Costas and V.N. Vakharia, 2005. Characterization of the nucleic acid-binding activity of the avian reovirus non-structural protein. NS J. Gen. Virol., 86: 1159-1169.

Wang, C.S. and S.L. Cheng, 2010a. Cloning and cDNA sequence analysis of the S3 gene of a novel duck reovirus strain $\mathrm{NP03}$ in China. J. Agric. Biot., 18: 567-572.
Wang, C.S. and S.L. Cheng, 2010b. Establishment and its application of RT-PCR for detection of novel duck reovirus. J. Agric. Biotechnol., 19: 388-392.

Xie, Q., J. Ji, T.T. Pickens, L. Du and Y. Cao et al., 2010. Rapid detection of infectious laryngotracheitis virus isolates by loop-mediated isothermal amplification. J. Virol. Methods, 165: 71-75.

$\mathrm{Xu}$, J. and Z. Zhang, 2009. Development of reversetranscription loop-mediated isothermal amplification for the detection of infectious bursal disease virus. J. Virol. Methods, 162: 267-271. 